\title{
Preamble: new development on advanced materials for photonics, sensing and energy applications
}

\author{
Bouchta Sahraoui • Yahia Boughaleb • \\ Anna Zawadzka • Asli Karakas
}

Published online: 4 December 2013

C Springer Science+Business Media New York 2013

The international conference devoted to new development on Advanced Materials for Photonics, Sensing and Energy Conversion Applications AMPSECA'2012 was supported initially by the newly created SMPM2A "Moroccan Association of Physics of Advanced Materials and their Applications" and organized jointly with this scientific association in El Jadida, Morocco by the University of Chouaib Doukkali, The University of Angers, The INSA of Lyon, The University of Valenciennes, the University of Lyon I.

This International Conference brought together leading researchers from different disciplines on one platform. This large gathering of academic and industrial researchers have provided in depth information on particular properties of materials and their potential applications. This event have focused on energy related issues and brought eminent scientists and program managers from various funding agencies in the form of plenary speakers and panel discussions.

B. Sahraoui $(\varangle)$

LUNAM Université, Université d'Angers, CNRS UMR 6200, Laboratoire

MOLTECH-Anjou, 2 bd Lavoisier, 49045 Angers Cedex, France

e-mail: bouchta.sahraoui@univ-angers.fr

URL: http://ead.univ-angers.fr/ sahraoui

Y. Boughaleb

Ecole Normale Supérieure, Université Hassan II Casablanca,

Casablanca, Morocco

e-mail: yboughaleb@yahoo.fr

\section{A. Zawadzka}

Faculty of Physics, Astronomy and Informatics, Institute of Physics, Nicolaus Copernicus University, Grudziadzka 5, 87-100 Torun, Poland e-mail: azawa@fizyka.umk.pl
A. Karakas
Department of Physics, Faculty of Sciences, Selcuk University,
Campus, Konya, Turkey
e-mail: akarakas@selcuk.edu.tr 
AMPSECA'2012, with more than 200 participants from various parts of the world, provides a unique opportunity for discussions on the most recent developments and future perspectives in the field of Advanced Materials for Photonics, Sensing and Energy Conversion Applications including biophotonics, by leading Moroccans Europeans and international researchers. This meeting aimed to stimulate better contacts between $\mathrm{PhD}$ students and senior scientists from all over the world and to create new opportunities for reinforcing or starting new international and multilateral collaborations.

The conference consisted of a total of 9 plenary lectures and 27 invited lectures and 40 oral contributions have been given. About 210 contributed papers have been presented in the poster forum. We have received 270 contributions from 30 countries.

The venues and dates of the conference 2012, as well as their organizing and scientific committees are listed below.

The International Conference on Advanced Materials for Photonics, Sensing and Energy Conversion Applications (AMPSECA 2012), December 5-7, 2012 El Jadida, Morocco Honorary Chair:

- Marian Marciniak (NIT-Warsaw, Poland)

Conference chairs:

- Yahia BOUGHALEB (Univ-El Jadida, Morocco)

- Bouchta SAHRAOUI (Univ-Angers, France)

Co-chairs:

- Mickaël LALLART (INSA-Lyon, France)

- Abdelowahed HAJJAJI (Univ-El Jadida, Morocco)

- Anne LERICHE (Univ-Valenciennes, France)

- Abdelhamid ERRACHID (Univ-Lyon I, France)

- Mina BAKASSE (Univ-El Jadida, Morocco)

- Abdezzahid ARBAOUI (Univ-El Jadida, Morocco)

- Khalil BENKHOUJA (Univ-El Jadida, Morocco)

Organizing Committee

Local Committee:

- Yahia BOUGHALEB (Univ-El Jadida, Morocco)

- Abdelowahed HAJJAJI (Univ-El Jadida, Morocco)

- Abdezzahid ARBAOUI (Univ-El Jadida, Morocco)

- Khalil BENKHOUJA (Univ-El Jadida, Morocco)

- Hassan OUAHMANE (Univ-El Jadida, Morocco)

- Mina BAKASSE (Univ-El Jadida, Morocco)

- Mohammed MONKADE (Univ-El Jadida, Morocco)

- Abdessamad EL BALLOUTI (Univ-El Jadida, Morocco)

International Committee:

- Bouchta SAHRAOUI (Univ-Angers, France)

- Yahia BOUGHALEB (Univ-El Jadida, Morocco)

- Daniel GUYOMAR (INSA-Lyon, France)

- Mickaël LALLART (INSA-Lyon, France)

- Nicole JAFFREZIK (Univ-Lyon I, France)

- Abdelhamid ERRACHID (Univ-Lyon I, France)

- Christian COURTOIS (Univ-Valenciennes, France) 
- Mohamed RGUITI (Univ-Valenciennes, France)

- Abdelkrim El-GHAYOURY (Univ-Angers, France)

- Abdelowahed HAJJAJI (Univ-El Jadida, Morocco)

- Anna ZAWADZKA (UMK, Torun, Poland)

- Ewa GONDEK (Cracow Univ. of Techn., Poland)

- Abdezahid ARBAOUI (Univ-El Jadida, Morocco)

- Khalil BENKHOUJA (Univ-El Jadida, Morocco)

- Mina BAKASSE (Univ-EL Jadida, Morocco)

Scientific Committee:

- Franck GASCOIN (univ- Caen, France)

- Daniel GUYOMAR (INSA-Lyon, France)

- Einar HALVORSEN (Univ-College-Vestfold, Norway)

- Oan BAUSELLS (Barcelona, Spain)

- Denis REMIENS (Univ-Lille, France)

- Yaroslav KORPAN (IMBG NASU, Ukrain)

- Peter MCBREEN (Univ-Laval, Québec, Canada)

- Adrien BADEL (Univ-Savoie, France)

- Nicole JAFFRIZIC (Univ-Lyon1, France)

- Rumyana BAKALSKA (Univ-Plovdiv, Bulgaria)

- Hafed BELMABROUK (Univ-Monastir, Tunisia)

- Karim BOUCHOUIT(University of Jijel, Jijel, Algeria)

- Fabrice CHARRA (CEA-Paris, France)

- Stelios COURIS (Univ-Patras, Greece)

- Beata DERKOWSKA (UMK-Torun, Poland)

- Jean EBOTHE (Univ-Reims, France)

- Abdelhamid ERRACHID (Univ-Lyon1, France)

- Adil FAIZ (Univ-Nancy, France)

- Maurizio FERRARI (CNR-IFN-Trento, Italy)

- Franciszek FIRSZT (UMK-Torun, Poland)

- Marc.D FONTANA (Univ-Metz, France)

- Andrzej GRAJA (Polish Acad. of Sciences Poland)

- Francoise GROLLEAU (Univ-Angers,UNAM, France)

- James G.GROTE (US Air Force Research, USA)

- Benoit GUIFFARD (Univ-Nantes, France)

- François KAJZAR (Univ-Angers, France)

- Aleksey KOLENDO (Univ-Kiev, Ukraine)

- Hafsa KORRI-YOUSSOUFI (Univ-Paris Sud, France)

- Mickaël LALLART (INSA-Lyon, France)

- Laurent LEBRUN (INSA-Lyon, France)

- lie LEFEUVRE (Univ-Paris Sud, France)

- Anne LERICHE (Univ-Valenciennes, France)

- Mustapha BOUSMINA (Acad HassanII, Morocco)

- Aurelia MEGHEA (Univ-Politehnica, Bucharest)

- Anna MIGALSKA-ZALAS(AJD-Czestochowa,)

- Andrzej MINIEWICZ (Univ-Wroclaw, Poland)

- Jaroslaw MYSZLIWIEC (Univ-Wroclaw, Poland)

- Sebastien Pruvost (INSA-Lyon, France)

- Ileana RAU (Univ-Bucharest, Romania) 
- Roberta RAMPONI (Politecnico Di Milano, Italy)

- Svatopluk CIVIS (Acad. of Sciences, Czech Republic)

- Jurek SADOWSKI (Upton-NY, USA)

- Marc SALLE (Univ-Angers, France)

- Zina SASSI (INSA-Lyon, France)

- Valery SERBEZOV (Univ-Plovdiv, Bulgaria)

- Anna ZAWADZKA (UMK-Torun, Poland)

- Mohammed ABID (Univ-Casablanca, Morocco)

- Mohammed ADDOU (Univ-kenitra, Morocco)

- Rachid AGOUNOUN (EST-Meknes, Morocco)

- Omar AMRANI (FST-Errachidia, Morocco)

- Abdezahid ARBAOUI (Univ-El Jadida, Morocco)

- Asmae ARBAOUI (Univ-Rabat, Morocco)

- Mimouna BAITOUL (Univ-Fez, Morocco)

- Mina BAKASSE (Univ-El Jadida, Morocco)

- Abdelfettah BARHDADI (ENS-Rabat, Morocco)

- Mohamed BELAICHE (Acad Hassan II, Morocco)

- Abdessamad EL BALLOUTI (ENSA-El Jadida, Morocco)

- Mohammed BENNAI (Univ-Casablanca, Morocco)

- Khalil BENKHOUJA (Univ-El Jadida, Morocco)

- Abdelillah BENYOUSSEF (Univ-Rabat, Morocco)

- Rachid EL BOUAYADI (Univ-Nador, Morocco)

- Brahim BOUBEKER (Univ-Ben M'Sik, Morocco)

- Khalid BOUZIANE (Univ-international Rabat,Morocco)

- Rajae CHERKAOUI (Acad. Hassan II, Morocco)

- Aouatif DEZAIRI (Univ-Casablanca, Morocco)

- El Mokhtar ESSASI (Acad Hassan II, Morocco)

- Mounir FAHOUME (Univ-Kénitra, Morocco)

- Ahmed QACHAOU (Univ -Kénitra, Morocco)

- Abdallah EL KENZ (Univ-Mohamed V, Morocco)

- Hamid EZZAHRAOUY (Univ-Mohamed V, Morocco)

- Mohamed LOULIDI (Univ-Mohamed V, Morocco)

- Reddad EL MOZNINE (Univ-EL Jadida, Morocco)

- Mohamed EL BOUZIANI (Univ-EL Jadida, Morocco)

- Mohammed FLIYOU (ENS-Casablanca, Morocco)

- Mustapha HADDAD (Univ-Meknes, Morocco)

- Mohamed EL HAFIDI (Univ-Ben M'Sik, Morocco)

- Abdelowahed HAJJAJI (Univ-El Jadida, Morocco)

- Mohamed HAMEDOUN (MASCIR-Rabat, Morocco)

- Bouchaib HARTITI (FST-Mohammedia, Morocco)

- Abdelillah KADDOURI (Univ-Marrakech, Morocco)

- Mohamed KEROUAD (Univ-Meknes, Morocco)

- Hassan LASSRI (Univ-Casablanca, Morocco)

- M'hamed MAZROUI (Univ-Hassan II, Morocco)

- Mohamed MONKADE (Univ-El Jadida, Morocco)

- Mohamed MOUSSETADE (Univ-Casablanca, Morocco)

- Rachid NASSIF (Univ-El Jadida, Morocco)

- Said OUASKIT (Univ-Casablanca, Morocco)

- Hassan OUAHMANE (ENSA-El Jadida, Morocco) 
- Mohammed OUAZZANI JAMIL (Univ-Fes, Morocco)

- Abderaouf RIDAH (Univ-Hassan II, Morocco)

- Mohammed SAHLAOUI (FST-Beni Mellal, Morocco)

- Hassan SAIDI (Acad Hassan II, Morocco)

- Dennon SAIFAOUI (Univ-Hassan II, Morocco)

- Mohammed SAJEDDINE (FST-Beni Mellal, Morocco)

- Abdallah ZRADBA (Univ-El Jadida, Morocco)

- Ewa GONDEK (Cracow Univ. of Techn., Poland)

- Salaheddine SAYOURI (Univ-Fès, Morocco)

Acknowledgments The Guest Editors Bouchta SAHRAOUI and Yahia BOUGHALEB on behalf of the organizers would like to acknowledge our main sponsor and partner OCP, and to thank the local authorities of EL Jadida (Province d'El Jadida et Conseil Régional de Abda Doukkala), IT Learning Academy, Master Tec, Scomédica, Hassan II Academy of sciences and Technology and Gasup for their Technical help and financial support. We would like also to thank Professor Marian Marciniak who is Editor in Chief of OQEL for his support, patience, collaboration and understanding. On behalf of Organizing Committee we express our thanks to all staff of Optical and Quantum Electronics. Professor Yahia BOUGHALEB and Dr Anna Zawadzka and Dr Asli Karakas would like to acknowledge Professor Bouchta SAHRAOUI for not only his support and help during the entire edition process of this special issue but also for his invitation as Guest Editors. On behalf of Organizing Committee we express our thanks to all the participants and to the authors and referees for their valuable contributions to the conference and the Proceedings of the AMPSECA'2012. We would like also to express our thanks to all colleagues and students for their help. 